\title{
On the Roles of "Stability" and "Convergence" in Semidiscrete Projection Methods for Initial-Value Problems
}

\author{
By Seymour V. Parter*
}

Abstract. Consider the initial value problem

$$
\begin{gathered}
\frac{d}{d t} u(t)=A u(t)+f(t), \quad t>0, \\
u(0)=u_{0},
\end{gathered}
$$

where $A$ is a linear operator taking $D(A) \subset X$ into $X$, where $X$ is a Banach space. Consider also semidiscrete numerical methods of the form: find $U_{N}(t):[0, T] \rightarrow X_{N}$ such that

$$
\begin{gathered}
\frac{d U_{N}}{d t}=A_{N} U_{N}+P_{N} f, \\
U_{N}(0)=U_{N}^{0} \in X_{N},
\end{gathered}
$$

where $X_{N}$ is a finite dimensional subspace and $P_{N}$ is a projector onto $X_{N}$.

The study of such numerical methods may be related to the approximation of semigroups and Laplace transform methods making use of the resolvent operators $(A-\lambda I)^{-1},\left(A_{N}-\lambda I_{N}\right)^{-1}$. The basic results require stability or weak stability and give convergence rates of the same order as in the steady state problems.

1. Introduction. In the early 1950 's, as scientists became concerned with the numerical solution of partial differential equations, there were many papers concerned with the questions of "stability" and "convergence" of solutions of difference approximations to time dependent problems (now called "equations of evolution"). ${ }^{* *}$ In 1951 M. A. Hyman, S. Kaplan and G. G. O’Brien [34] discussed this question and described the von Neumann "stability criterion". In the same year W. Leutert [32] gave an example of an "unstable" scheme which, nevertheless, was in some sense "convergent". These results were followed by many, many convergence proofs ([12], [24], [25], [26] for a few). In 1956 there appeared a paper by Jim Douglas, Jr. [11] "On the Relation Between Stability and Convergence in the Numerical Solution of Linear Parabolic and Hyperbolic Differential Equations." However, Douglas was distracted by

Received June 29, 1978; revised May 1, 1979.

AMS (MOS) subject classifications (1970). Primary 65M10, 65M15.

*Supported by the Office of Naval Research under Contract No. N00014-76-C-0341.

**No attempt at describing the history of this subject could possibly be complete. I make no claim that the above discussion is a complete description of the early pioneering papers. At the same time, no discussion of this topic can even begin without mentioning the fundamental paper by R. Courant, K. O. Friedrichs and H. Lewy [9]. 
Leutert's example and restricted his efforts to the proof that stability (under appropriate "consistency" conditions) led to convergence. In the same year (1956) the fundamental paper of P. Lax and R. D. Richtmyer [30] employed the principle of uniform boundedness to show that if one demanded convergence for a sufficiently broad class of problems, then stability and convergence are indeed equivalent. This result is the famous "Lax Equivalence Theorem." In 1958 H. F. Trotter*** [43] returned to the questions raised by Lax and Richtmyer and put the results (and theory) into the framework of the theory of Linear Semi-groups.

During this time an effort was made to understand and clarify the several possible definitions of "stability." In particular, in 1960 Strang [40] discussed "weak stability" in which the solution operator becomes unbounded as $\Delta t \rightarrow 0$ but at a rate which is $O\left(\Delta t^{-P}\right)$. He proved the following beautiful theorem: If the solution $u(x, t)$ is sufficiently smooth, then the discrete solution $U(x, t, \Delta t)$ of such a weakly stable method is convergent to $u(x, t)$ and the "rate of convergence" is that predicted by the truncation error. In $1962 \mathrm{H}$. O. Kreiss [29] wrote a definitive paper on the relationship between various notions of stability, the von Neumann Criterion and the concept of "Properly Posed in the Sense of Petrowsky" (see Aronson [1], Wendroff [44] also).

But here we are, some twenty years later, and most research in numerical methods for partial differential equations is not concerned with difference methods. The interest is now on Ritz-Galerkin methods, collocation methods, and in general "Projection Methods." And, as one reads the present day literature one rarely sees the word "stability." There are many, many "convergence" theorems (with appropriate smoothness assumptions).

Of course, there is a good reason for this state of affairs. Most Ritz-Galerkin methods with a continuous time variable are automatically stable. In fact, this observation is the beginning and the motivation for the paper by B. Swartz and B. Wendroff [42]-one of the early "American" papers on the subject of Galerkin methods for time dependent problems. Moreover, much of the research of today is concerned with a host of immediate questions, e.g. time discretization by multistep methods (see $[2],[6],[10],[52]$ for a few), replacement of integration by quadrature methods (see [17], [37]), collocation (see [8], [15], [48]).

Nevertheless, particularly as we begin to look at more sophisticated projection methods, e.g. collocation, it seems reasonable to look again at this concept of "stability" and its relationship to "convergence."

In Section 2 we formulate the problem of equations of evolution and semidiscrete numerical methods based on a sequence of subspaces $\left\{X_{n}\right\}$ and related projection operators $\left\{P_{n}\right\}$.

In Section 3 we discuss some examples. In Section 4 we use a modification of a now standard proof of the "Trotter Approximation Theorem" to discuss the roles of stability and convergence in a general setting.

*** This famous paper is particularly interesting. Most numerical analysts do not realize that it is primarily devoted to the stability-convergence question, and, most probabilists, who-if they have read the paper-must know, seldom (if ever) mention this fact. 
This discussion explicitly shows how the semigroup theory clarifies much of the existing literature. In this connection, it is appropriate to mention that Helfrich [21] and Fujita and Mizutani [16] make explicit use of the theory of holomorphic semigroups in their treatment of parabolic problems.

In Section 5 we discuss a particular definition of "weak stability" and show how one may obtain "convergence theorems" with such methods provided one has some additional smoothness and makes a particular choice of "initial values." The results of this section may be regarded as analogs of the theorem of Strang.

These results of Section 5 are also closely related to results of Beals [3] for the partial differential equation.

In Section 6 we discuss parabolic problems in one space dimension.

2. The Problem. Let $X$ be a Banach Space and let $A$ be a densely defined linear operator from $D(A) \subset X$ into $X$. We are concerned with "semidiscrete" numerical methods for the approximate solution of the initial-value problem

$$
\left\{\begin{array}{l}
\frac{d}{d t} u(t)=A u(t)+f(t), \quad t>0, \\
u(0)=u_{0} \in X
\end{array}\right.
$$

where $f(t)$ is an $X$-valued function of $t$. By a solution (see [22, p. 619], [35, p. 105]) we mean an $X$-valued function $u(t)$ which is

(i) continuous for $t \geqslant 0$,

(ii) continuously differentiable (in $t$ ) for $t>0$;

moreover,

(iii) for $t>0, u(t) \in D(A)$, and

(iv) Eqs. (2.1) are satisfied.

We assume that Eqs. (2.1) describe a "properly posed problem." To be more precise, we assume:

H.1: $A$ is the infinitesimal generator of a $C_{0}$ semigroup $T(t)$, and, the unique solution of (2.1) is given by

$$
u(t)=T(t) u_{0}+\int_{0}^{t} T(t-s) f(s) d s .
$$

Moreover, the semigroup, $T(t)$, satisfies

$$
\|T(t)\| \leqslant M e^{\omega t}
$$

where $M>0, \omega \geqslant 0$ are fixed constants.

A related problem is the "steady state" or time independent problem

$$
A u+f_{0}=0 \text {, }
$$

where $f_{0}$ is a fixed element of $X$.

We assume that this problem has a unique solution $u$ for all $f_{0} \in X$. In fact, we assume:

H.2: $A^{-1}$ exists as a bounded linear operator defined on all of $X$. Moreover, 
the "resolvent condition" is satisfied, i.e., there is a constant $M$ such that, for all real $\lambda>0,(A-\lambda I)^{-1}$ exists as a bounded linear operator defined on all of $X$ and

$$
\left\|(A-\lambda I)^{-n}\right\| \leqslant M \lambda^{-n}
$$

Remark. Assumption H.1 implies an estimate of the form of (2.5). Conversely, under appropriate assumptions on $f(t)$, assumption H.2 implies H.1. See [35, p. 21].

A large class of numerical methods for the approximate solution of the steady state problem (2.4) are described in the following manner.

Let $\left\{X_{n}\right\}_{1}^{\infty}$ be a family of finite dimensional subspaces of $X$. (For convenience, let $\operatorname{dim} X_{n}=n$.) Let $\left\{P_{n}\right\}_{1}^{\infty}$ be an associated family of uniformly bounded projections of $X$ onto $X_{n}$ with

$$
\left\|P_{n}\right\| \leqslant M_{0}
$$

Let $\left\{A_{n}\right\}_{1}^{\infty}$ be an associated family of nonsingular maps from $X_{n}$ onto $X_{n}$. The approximant $u_{n} \in X_{n}$ satisfies the equation

$$
A_{n} u_{n}=-P_{n} f_{0}
$$

In fact, the Galerkin method (or, the direct projection method) is obtained when

$$
X_{n} \subset D(A)
$$

and

$$
A_{n}=P_{n} A
$$

A typical theorem associated with the above type of approximation scheme takes the following form.

THEOREM T. There exists a Banach space $\widetilde{X} \subset X$ with

$$
\|y\| \leqslant C\|y\|_{\widetilde{X}}
$$

and a function $F(n) \downarrow 0$ as $n \rightarrow \infty$ such that: if $u$, the solution of (2.4), is in $\widetilde{X}$, then

$$
\left\|u-u_{n}\right\| \leqslant F(n)\|u\|_{\tilde{X}}
$$

If we define $Q_{n}$ on $D(A)$ by

$$
Q_{n}=A_{n}^{-1} P_{n} A
$$

we can restate Theorem $\mathrm{T}$ (T for "typical") as:

Let $u \in D(A) \cap \widetilde{X}$; then

$$
\left\|Q_{n} u-u\right\| \leqslant F(n)\|u\|_{\tilde{X}}
$$

Once one has developed this procedure for the steady state problem (1.4) and obtained Theorem T, one is naturally led to consider the following "continuous time, semidiscrete numerical method" for (2.1): Find a $X_{n}$-valued function $u_{n}(t)$ which is

(i) continuous for $t \geqslant 0$,

(ii) continuously differentiable for $t>0$, 
and satisfies the initial value problem

$$
\left\{\begin{array}{l}
\frac{d}{d t} u_{n}(t)=A_{n} u_{n}(t)+P_{n} f(t), \quad t>0 \\
u_{n}(0)=U_{0, n} \in X_{n}
\end{array}\right.
$$

where $U_{0, n}$ is chosen in some prescribed way so that $\left\|u_{0}-U_{0, n}\right\|$ is small.

In fact, there are two methods for choosing $U_{0, n}$ which come to mind at once. These are

$$
U_{0, n}=P_{n} u_{0}
$$

and, if $u_{0} \in D(A)$,

$$
U_{0, n}=Q_{n} u_{0}
$$

Since $A_{n}$ is a linear map from $X_{n}$ to $X_{n}$, and since $X_{n}$ is of finite dimension, each $A_{n}$ generates a $C_{0}$ semigroup $S_{n}(t): X_{n} \rightarrow X_{n}$ given by

$$
S_{n}(t)=e^{A} n^{t}
$$

Moreover, the solution of $(2.11)$ is given by

$$
u_{n}(t)=S_{n}(t) U_{0, n}+\int_{0}^{t} S_{n}(t-s) P_{n} f(s) d s
$$

Definition 2.1. The semidiscrete method described by (2.11) is "stable" if there exist constants $\bar{M}, \bar{\omega}$ (independent of $n$ ) such that

$$
\left\|S_{n}(t)\right\| \leqslant \bar{M} e^{\bar{\omega} t}
$$

Remark. This definition of "stable" is classical and was introduced by Lax and Richtmyer [30] and Trotter [43]. The "norm" used in (2.14) is the norm of $X$ restricted to $X_{n}$.

Applying the general theory of semigroups, we find that the semidiscrete method is stable if and only if there is a constant $M_{1}$ such that, for all real $\lambda>\bar{\omega}$ we have

$$
\left\|\left(A_{n}-\lambda I\right)^{-m}\right\| \leqslant \frac{M_{1}}{(\lambda-\bar{\omega})^{m}}, \quad m=1,2, \ldots
$$

Unfortunately, (2.15) is an infinite system of estimates and, in general, not easy to verify. A much stronger result is: the semigroups $S_{n}(t)$ satisfy

$$
\left\|S_{n}(t)\right\| \leqslant e^{\bar{\omega} t}
$$

if and only if for every real $\lambda>\bar{\omega}$ we have

$$
\left\|\left(A_{n}-\lambda I\right)^{-1}\right\| \leqslant \frac{1}{(\lambda-\bar{\omega})}
$$

In many cases we find that the semigroup $T(t)$ is not only a semigroup in $X$, 
but also is a semigroup in $\widetilde{X}$. For this reason we will sometimes find it convenient to assume

H.3: There are constants $M_{2}$ and $\alpha$ such that if $x \in \widetilde{X}$, then $T(t) x \in \widetilde{X}$ and

$$
\|T(t) x\|_{\widetilde{X}} \leqslant M_{2} e^{\alpha t}\|x\|_{\widetilde{X}}
$$

We close this section with the observation that stable semidiscrete methods are "stable" under bounded perturbation. Specifically, we have the following

THEOREM 2.1. Suppose the semidiscrete method described by (2.11) is stable. Let $\left\{B_{n}\right\}$ be a family of uniformly bounded linear operators from $X_{n}$ to $X_{n}$

$$
B_{n}: X_{n} \rightarrow X_{n}
$$

and there is a constant $B$ such that

$$
\left\|B_{n}\right\| \leqslant B
$$

Consider the semidiscrete system

$$
\left\{\begin{array}{l}
\frac{d v_{n}}{d t}=\left(A_{n}+B_{n}\right) v_{n}+P_{n} f, \quad t>0 \\
v_{n}(0)=V_{n, 0} \in X_{n}
\end{array}\right.
$$

Then this semidiscrete method is stable.

Proof. It suffices to consider the homogeneous case, i.e., $f \equiv 0$. Since (2.19) is a linear system of ordinary differential equations with constant coefficients, there is a solution $v_{n}(t)$. Moreover, we may write

$$
v_{n}(t)=S_{n}(t) V_{n, 0}+\int_{0}^{t} S_{n}(t-s) B_{n} v_{n}(s) d s
$$

The theorem now follows from Gronwall's Inequality; see [4] and the basic estimate (2.14).

3. Examples. Before proceeding to the development of the general theory, we present some examples which are of particular interest.

Example 1. Let $\Omega$ be a smooth domain in $R_{n}$. Let

$$
\begin{gathered}
X=L^{2}(\Omega), \\
A=\Delta=\sum_{j=1}^{n} \frac{\partial^{2}}{\partial x_{j}^{2}}
\end{gathered}
$$

and

$$
D(A) \equiv\left\{u \in X ; u \in \stackrel{\circ}{H}_{1}(\Omega) \cap H_{2}(\Omega)\right\} .
$$

Let $X_{n} \subset D(A)$ be chosen so as to satisfy certain approximation properties (as in [2], [7] , [14]). Let $P_{n}$ denote $L^{2}$ projection onto $X_{n}$. Let 


$$
A_{n}=P_{n} A \text {. }
$$

In this case we are dealing with Galerkin's Method for the classical Dirichlet problem. A typical result (Theorem $\mathrm{T}$ ) takes the form: Let

$$
\widetilde{X} \equiv\left\{u \in D\left(A^{k}\right)\right\}
$$

where $k>1$ is an integer. Let

$$
\|u\|_{\tilde{X}}^{2}=\sum_{j=0}^{k}\left\|A^{j} u\right\|^{2}
$$

Then, for $u \in \widetilde{X}$, we have

$$
\left\|Q_{n} u-u\right\|=\left\|A_{n}^{-1} P_{n} A u-u\right\| \leqslant F(n)\|u\|_{\tilde{X}} .
$$

See [7], [21], [51]. Turning to the parabolic problem

$$
\left\{\begin{array}{l}
\frac{\partial u}{\partial t}=\Delta u=A u, \quad t>0 \\
u(x, 0)=u_{0}(x),
\end{array}\right.
$$

we see that it is relatively easy to show that the semidiscrete procedure is stable. In fact, we have: if $u_{n}(t) \in X_{n}$ satisfies

$$
\left\{\begin{array}{l}
\frac{\partial u_{n}}{\partial t}=P_{n} \Delta u_{n}, \quad t>0 \\
u_{n}(x, 0)=U_{n, 0}(x) \in X_{n},
\end{array}\right.
$$

then after multiplication by $u_{n}(t)$ we obtain

$$
\frac{1}{2} \frac{d}{d t}\left\|u_{n}(t)\right\|^{2}=\left(u_{n}, P_{n} \Delta u_{n}\right)=\left(u_{n}, \Delta u_{n}\right) \leqslant 0
$$

Hence $\left\|u_{n}(t)\right\| \leqslant\left\|U_{n, 0}\right\|$, which implies that $\left\|S_{n}(t)\right\| \leqslant 1$. Thus, one easily obtains results of the form: if $u(x, t)$ and $u_{t}(x, t) \in \widetilde{X}$, then

$$
\left\|u(\cdot, t)-u_{n}(\cdot, t)\right\| \leqslant C(t) F(n) \max _{0 \leqslant s \leqslant t}\left[\|u(\cdot, s)\|_{\tilde{X}}+\left\|u_{t}(\cdot, s)\right\|_{\widetilde{X}}\right]
$$

See [7], [13], [36].

Finally, in this case, the basic hypotheses H.1, H.2 and H.3 all hold.

Example 2. Choose $X$ and $A$ as in the previous example. However, we now require only that the subspaces $X_{n} \subset X$ belong to $H_{1}(\Omega)$. Let (, ) denote the inner product in $L^{2}(\Omega)$ and $\langle$,$\rangle denote the inner product in L^{2}(\partial \Omega)$. The numerical method for the steady state problem

$$
\Delta U+f=0
$$

takes the form: find $u_{n} \in X_{n}$ such that 


$$
\left(\nabla u_{n}, \nabla v_{n}\right)+\left(f, v_{n}\right)+n^{\sigma}\left\langle u_{n}, v_{n}\right\rangle=0 \text { for all } v_{n} \in X_{n} .
$$

Here $\sigma$ is a positive constant. In this case we are dealing with the "penalty" method for the Dirichlet problem. The appropriate $P_{n}$ is again the $L^{2}$ projection onto $X_{n}$. However, the operator $A_{n}$ is a perturbation of the Galerkin operator. This problem has been analyzed under appropriate conditions on $X_{n}$; see [7].

Our next example is one of particular interest from the point of view of the questions raised in this report. Convergence theorems have been proven by J. Douglas and T. Dupont [15] and by J. H. Cerutti and S. V. Parter [8]. However, these authors have not touched on the questions of stability in the appropriate norm.

Example 3. Let $X=C[0,1] \cap\{u ; u(0)=u(1)=0\}$. Let

$$
A=\left(\frac{d}{d x}\right)^{2}+a(x) \frac{d}{d x}+c(x)
$$

where

$$
c(x)<0
$$

and $a(x)$ is a smooth function. Let $0=x_{0}<x_{1}<\cdots<x_{m}=1$ and let $I_{j}=$ $\left[x_{j-1}, x_{j}\right]$. Let $k$ be a fixed positive integer, and let

$$
X_{n} \equiv\left\{u(x) \in X \cap C^{1}[0,1] ;\left.u\right|_{I_{j}} \in P_{k+2}, j=1,2, \ldots, m\right\}
$$

where $P_{k+2}$ denotes the polynomials of degree $<k+2$, i.e. of "order" $k+2$. Let $\xi_{1}, \ldots, \xi_{k}$ be the Gaussian points on $[0,1]$ (see [8] or [15] for a more complete discussion) and let

$$
\xi_{j s}=x_{j-1}+\xi_{s}\left(x_{j}-x_{j-1}\right), \quad j=1, \ldots, m, s=1, \ldots, k,
$$

be the local Gauss points. The collocation method for the steady state problem studied by de Boor and Swartz [5] (their work is far more general, but this is the case of interest here) is described by the following procedure. Find $u_{n} \in X_{n}$ such that

$$
\left(A u_{n}\right)\left(\xi_{j s}\right)=f\left(\xi_{j s}\right), \quad j=1, \ldots, m, s=1, \ldots, k \text {. }
$$

For the parabolic problem

$$
\left\{\begin{array}{l}
\frac{\partial u}{\partial t}=A u+f(x, t) \\
u(x, 0)=u_{0}(x) .
\end{array}\right.
$$

The collocation method takes the following form: find $u_{n}(x, t) \in X_{n}$ (for each fixed t) such that

$$
\begin{gathered}
\frac{\partial u_{n}}{\partial t}\left(\xi_{j s}, t\right)=\left(A u_{n}\right)\left(\xi_{j s}, t\right)+f\left(\xi_{j s}, t\right), \quad j=1,2, \ldots, m, s=1, \ldots, k \\
u_{n}(x, 0)=U_{n, 0}(x) \in X_{n}
\end{gathered}
$$


Both Dupont and Douglas [15] and Cerutti and Parter [8] showed that one obtains the same kind of error estimates for the parabolic problem as de Boor and Swartz [5] obtained for the elliptic (steady state problem) when one used

$$
U_{n, 0}(x)=Q_{n} u_{0} .
$$

Those results showed convergence in the maximum norm. Yet none of these authors established stability in the maximum norm. In terms of the discussion of this report, Dupont and Douglas established stability in the $H_{1}$ norm and used the imbedding of $H_{1}[0,1]$ in $C[0,1]$ to establish convergence in the presence of sufficient smoothness. On the other hand, Cerutti and Parter established a certain "resolvent estimate" which (i) came from the $H_{1}$ stability and (ii) could be interpreted as a form of weak stability and (iii) was good enough to allow the use of the Laplace transform in the case of smooth solutions. As far as this author knows, the question of "maximum norm stability" for this collocation scheme is still an open problem.

Our next example shows that the validity of Theorem $T$ (for the steady state case) does not imply the stability or general convergence of the time dependent numerical method. In this example the operator $A_{n}$ is a perturbation of the Galerkin operator. Moreover, in this case $\widetilde{X}=X$.

Example 4. Let $X=L^{2}[0, \pi]$. Let

$$
A=(d / d x)^{2}
$$

with

$$
D(A)=\left\{u \in H_{2}(0, \pi) ; u(0)=u(\pi)=0\right\} .
$$

Let

$$
\begin{gathered}
X_{n}=\operatorname{span}\{\sin j x\}_{j=1}^{n}, \\
Y_{n}=\operatorname{span}\{\sin n x\}
\end{gathered}
$$

We write

$$
X_{n}=X_{n-1} \oplus Y_{n}
$$

and let

$$
A_{n}=\left[\left(\frac{d}{d x}\right)^{2}\right] \oplus\left[-\left(\frac{d}{d x}\right)^{2}\right] .
$$

Of course $P_{n}$ is the $L^{2}$ projection onto $X_{n}$. If $u \in D(A)$ and $A u=f$ with

$$
f \sim \sum_{j=1}^{\infty} f_{j} \sin j x \in L^{2}(0, \pi),
$$

then

$$
u=\sum_{j=1}^{\infty}\left(-f_{j} / j^{2}\right) \sin j x
$$


Clearly,

$$
P_{n} f=\sum_{j=1}^{n} f_{j} \sin j x
$$

and the solution of $A_{n} u_{n}=P_{n} f$ is given by

$$
u_{n}(x)=\sum_{j=1}^{n-1}\left(-f_{j} / j^{2}\right) \sin j x+\frac{f_{n}}{n^{2}} \sin n x
$$

We have the easy error estimate

$$
\left\|u-u_{n}\right\|^{2}=\frac{4\left|f_{n}\right|^{2}}{n^{4}}+\sum_{j=n+1}^{\infty}\left|f_{j}\right|^{2} / j^{4} \leqslant 4 \sum_{j=n}^{\infty} \frac{\left|f_{j}\right|^{2}}{j^{4}} \leqslant \frac{4\|f\|^{2}}{n^{4}} \rightarrow 0 .
$$

On the other hand, let $u_{0}=\sin n x$. Then

$$
S_{n}(t) u_{0}=e^{n^{2}} u_{0}
$$

Thus, the semidiscrete method for the initial-value problem

$$
\left\{\begin{array}{l}
\frac{\partial u}{\partial t}=A u \\
u(x, 0)=u_{0}
\end{array}\right.
$$

is not stable in any norm!!

In Example 4 we are dealing with a perturbation of Galerkin's method (see (2.8a), (2.8b)). In our next example we have a direct projection method which appears to be unstable.

Example 5. Let $0<\nu<1$, and let

$$
A=\left[\begin{array}{ll}
\nu & 1 \\
1 & \nu
\end{array}\right]
$$

Let $u(x, t)=\left[u_{1}(x, t), u_{2}(x, t)\right]^{T}$ and consider the mixed initial-value boundary value problem

$$
\begin{aligned}
& u_{t}=A u_{x}, \quad 0 \leqslant x \leqslant 1, \\
& u(x, 0)=u_{0}(x), \\
& u_{1}(0, t)=u_{1}(1, t)=0, \quad t>0 .
\end{aligned}
$$

In [20] Max D. Gunzburger considered the following semidiscrete Galerkin approach to this problem.

Let $X_{m}$ be determined by using cubic $B$-splines on a uniform mesh with $u_{1}^{m}(0)$ $=u_{1}^{m}(1)=0$ and $u_{2}^{m}(0), u_{2}^{m}(1)$ unspecified. The semidiscrete equations are obtained by requiring

$$
\left(u_{t}^{m}-A u_{x}^{m}, v^{m}\right)=0 \quad \text { for every } v^{m} \in X_{m} .
$$


In his interesting report [20] Gunzburger asserts that computational results indicate instability. He discusses the possible reasons for these difficulties.

If there is instability, as the computations suggest, we have here an example of a direct projection method which is unstable.

4. The Basic Results. In this section we prove the general theorems which are essentially restatements of the Lax-Richtmyer-Trotter results in our present context. The main result is that for stable semidiscrete numerical methods of the form described by (2.11) we can "lift" the results of Theorem T.

Our first result is a modification and interpretation of a basic identity which is usually used in the proof of the Trotter Approximation Theorem (see Pazy [35]).

Lemma 4.1. For every $x \in X$ we have $(t>0)$

$$
\begin{aligned}
A_{n}^{-1}\left[P_{n} T(t)-S_{n}(t) P_{n}\right] A^{-1} x \\
=\int_{0}^{t} S_{n}(t-s)\left[A_{n}^{-1} P_{n}-P_{n} A^{-1}\right] T(s) x d s .
\end{aligned}
$$

Proof. Let $t>0$ be fixed and let

$$
G_{n}(s)=S_{n}(t-s) A_{n}^{-1} P_{n} T(s) A^{-1} x .
$$

Then $G_{n}(s)$ is a differentiable function of $s, 0<s<t$. Using the basic relations

$$
\begin{gathered}
T(t) A z=A T(t) z, \quad \text { for } z \in D(A), \\
\frac{d}{d t} T(t) z=A T(t) z, \quad \text { for } z \in X, t>0, \\
A_{n} S_{n}(t)=S_{n}(t) A_{n}, \quad \text { in } X_{n}, \\
\frac{d}{d t} S_{n}(t) z=A_{n} S_{n}(t) z, \quad \text { for } z \in X_{n},
\end{gathered}
$$

we find that

$$
\frac{d}{d s} G_{n}(s)=S_{n}(t-s)\left[A_{n}^{-1} P_{n}-P_{n} A^{-1}\right] T(s) x
$$

Integrating this last relationship from 0 to $t$ yields (4.1).

LEMMA 4.2. For every $u \in D\left(A^{2}\right)$ we have

$$
Q_{n} T(t) u-S_{n}(t) Q_{n} u=\int_{0}^{t} S_{n}(t-s) P_{n}\left[Q_{n} T(s) A u-T(s) A u\right] d s .
$$

Proof. Let $x=A^{2} u$ and apply (4.1).

For the moment, we restrict our attention to the case $f(t) \equiv 0$.

TheOREM 4.1. Suppose $f(t) \equiv 0$. Suppose H.1 and H.2 hold. Suppose that Theorem $\mathrm{T}$ holds and the semidiscrete method is stable, i.e. (2.14) holds. Let $u(t)$ be the solution of (2.1). Let $u_{n}(t)$ be the solution of (2.11) with $U_{n, 0}$ given by $(2.12 \mathrm{~b})$. Let $u(t)$ and $A u(t)=d u(t) / d t$ belong to $D(A) \cap \widetilde{X}$. Then 


$$
\left\|Q_{n} u(t)-u_{n}(t)\right\| \leqslant \bar{M} M_{0} F(n) \int_{0}^{t} e^{\bar{\omega}(t-s)}\|A u(s)\|_{\tilde{X}} d s
$$

and

$$
\left\|u(t)-u_{n}(t)\right\| \leqslant F(n)\left[\|u(t)\|_{\widetilde{X}}+\bar{M} M_{0} \int_{0}^{t} e^{\bar{\omega}(t-s)}\|A u(s)\|_{\widetilde{X}} d s\right]
$$

Proof. Apply Lemma 4.2 and Theorem T (under the integral sign) to obtain (4.3a). Then (4.3b) follows from the triangle inequality and Theorem $T$.

TheOREM 4.2. Suppose $f(t) \equiv 0$. Suppose H.1, H.2 and H.3 hold and the semidiscrete method is stable. Suppose Theorem $\mathrm{T}$ holds. Let $u(t)$ be the solution of (2.1) and $u_{n}(t)$ be the solution of (2.11) with $U_{n, 0}$ given by (2.12b). Let $u_{0}$ and $A u_{0}$ belong to $D(A) \cap \widetilde{X}$. Then

$$
\begin{aligned}
\left\|Q_{n} u(t)-u_{n}(t)\right\| & \leqslant \bar{M} M_{0} M_{2} F(n)\left[\int_{0}^{t} e^{\bar{\omega}(t-s)} e^{\alpha s} d s\right]\left\|A u_{0}\right\|_{\tilde{X}} \\
& \leqslant\left(\bar{M} M_{0} M_{2}\right) C(t) F(n)\left\|A u_{0}\right\|_{\tilde{X}}
\end{aligned}
$$

where

$$
C(t)=\left[\int_{0}^{t} e^{\bar{\omega}(t-s)} e^{\alpha s} d s\right]
$$

Moreover,

$$
\left\|u_{n}(t)-u(t)\right\| \leqslant F(n)\left[M_{2} e^{\alpha t}\left\|u_{0}\right\|_{\widetilde{X}}+\left(\bar{M} M_{0} M_{2}\right) C(t)\left\|A u_{0}\right\|_{\widetilde{X}}\right] .
$$

Proof. Apply H.3 to (4.3a) and (4.3b) in Theorem 4.1.

Remark. Note the differences in the hypotheses of Theorems 4.1 and 4.2. In Theorem 4.2 we assume $u_{0}$ and $A u_{0} \in D(A) \cap \widetilde{X}^{\dagger}{ }^{\dagger}$ which is the hypothesis of Theorem 4.1 .

Definition 4.1. The semidiscrete method described by (2.11) is "convergent" if for all $u_{0} \in X$ and all $\tau>0$ we have

$$
\max \left\{\left\|S_{n}(t) U_{n, 0}-T(t) u_{0}\right\| ; 0<t<\tau\right\} \rightarrow 0 \text { as } n \rightarrow \infty
$$

whenever

$$
\left\|U_{0, n}-u_{0}\right\| \rightarrow 0 \text { as } n \rightarrow \infty
$$

THEOREM 4.3. Let

$$
V \equiv\{u \in D(A) \cap \widetilde{X} ; A u \in D(A) \cap \widetilde{X}\} .
$$

Suppose H.1, H.2, H.3 hold. Suppose Theorem T holds and the semidiscrete method is stable. Suppose $V$ is dense in $X$. Then, the semidiscrete method is convergent.

Proof. Let $u_{0} \in X$. Let $\left\{v^{(k)}\right\}_{k=1}^{\infty}$ be a sequence satisfying

\footnotetext{
${ }^{\dagger}$ Hence, by $H .3 u(t)$ and $A u(t)$ belong to $D(A) \cap \tilde{X}$.
} 
(i) For every $k, v^{(k)} \in V$,

(ii) $\left\|v^{(k)}-u_{0}\right\| \rightarrow 0$ as $k \rightarrow \infty$.

Then for every $k$ we have

$$
\begin{aligned}
& \left\|S_{n}(t) U_{0, n}-T(t) u_{0}\right\| \\
& \quad \leqslant\left\|S_{n}(t)\left[U_{0, n}-Q_{n} v^{(k)}\right]\right\|+\left\|S_{n}(t) Q_{n} v^{(k)}-T(t) v^{(k)}\right\|+\left\|T(t)\left[v^{(k)}-u_{0}\right]\right\| .
\end{aligned}
$$

Given $\epsilon>0$ we may choose $k_{0}$ so large that

$$
\left(1+M+\bar{M} e^{\bar{\omega} \tau}\right)\left\|u_{0}-v^{\left(k_{0}\right)}\right\| \leqslant \epsilon / 10 .
$$

Then

$$
\begin{aligned}
& \left\|S_{n}(t)\left[U_{0, n}-Q_{n} v^{\left(k_{0}\right)}\right]\right\| \\
& \quad \leqslant \bar{M} e^{\bar{\omega} t}\left\|U_{0, n}-u_{0}\right\|+\bar{M} e^{\bar{\omega} t}\left\|u_{0}-v^{\left(k_{0}\right)}\right\|+\bar{M} e^{\bar{\omega} t}\left\|v^{\left(k_{0}\right)}-Q_{n} v^{\left(k_{0}\right)}\right\| .
\end{aligned}
$$

Thus, employing Theorem 4.2 with $k_{0}$ fixed, we have

$$
\lim _{n \rightarrow \infty}\left\|S_{n}(t) Q_{n} v^{\left(k_{0}\right)}-T(t) v^{\left(k_{0}\right)}\right\|=0
$$

and

$$
\lim \sup \left\|S_{n}(t) U_{0, n}-T(t) u_{0}\right\| \leqslant \epsilon / 10 .
$$

Hence, the theorem is proven.

Employing the "Principle of Uniform Boundedness" in what is now a familiar argument (see [30], [38]), we obtain the converse result.

ThEOREM 4.4. Suppose H.1 and H.2 hold. Suppose the semidiscrete method is convergent. Then the method is stable.

Returning to the general case when $f(t) \not \equiv 0$, we recall that $\mathrm{H} .1$ includes the assumption that (2.1) has a solution $u(t)$; and this solution is given by (2.2).

TheOREM 4.5. Assume that H.1, H.2, H.3 hold. Assume that Theorem T holds and the semidiscrete method is stable. Let $u(t)$ be the solution of $(2.1)$ while $u_{n}(t)$ is the solution of (2.11). Let $u_{0}=U_{n, 0}=0$. Suppose that

$$
f(t) \in D(A) \cap \widetilde{X}, \quad A f(t) \in D(A) \cap \widetilde{X}
$$

Let $C(t)$ be given by (4.4b). Then

$$
\begin{aligned}
\left\|Q_{n} u(t)-u_{n}(t)\right\| \leqslant & \int_{0}^{t}\left(\bar{M} M_{0} M_{2}\right) F(n) C(t-s)\|A f(s)\|_{\widetilde{X}} d s \\
& +\int_{0}^{t} \bar{M} e^{\bar{\omega}(t-s)}\left\|\left(Q_{n}-P_{n}\right) f(s)\right\|_{X} d s
\end{aligned}
$$


Proof. We have

$$
Q_{n} u(t)-u_{n}(t)=\int_{0}^{t}\left[Q_{n} T(t-s)-S_{n}(t-s) P_{n}\right] f(s) d s .
$$

That is,

$$
\begin{aligned}
Q_{n} u(t)-u_{n}(t)= & \int_{0}^{t}\left[Q_{n} T(t-s)-S_{n}(t-s) Q_{n}\right] f(s) d s \\
& +\int_{0}^{t} S_{n}(t-s)\left[Q_{n}-P_{n}\right] f(s) d s .
\end{aligned}
$$

Thus, the theorem follows from Theorem 4.2.

Of course, one can now go on to assume that $f$ is approximated by functions $f^{(k)} \in V$. In this way one obtains general convergence proofs similar to Theorem 4.3 for the general case.

5. Weak Stability and the Laplace Transform. In the finite difference case, where the approximate solution is defined only at times $t_{k}=k \Delta t$, one sometimes defines "weak stability" by the condition (see [19], [29])

$$
\left\|S_{n}\left(t_{k}\right)\right\| \leqslant \bar{M} e^{\bar{\omega} t} k(\Delta t)^{-P}, \quad k=1,2, \ldots,
$$

where $P$ is a fixed positive number. In analogy to this one might consider in the semidiscrete case a definition of weak stability by the condition

$$
\left\|S_{n}(t)\right\| \leqslant \bar{M} e^{\bar{\omega} t} n^{P}, \quad t>0
$$

where we remember that $n=\operatorname{dim} X_{n}$.

Unfortunately, at this time we have not seen how to effectively study condition (5.1). Thus, for our purposes it is useful to work with the resolvent conditions (2.15), (2.16b) as the basis of stability and a corresponding concept of "weak stability".

Definition 5.1. The semidiscrete method described by (2.11) is "weakly stable" if there exists a function $M_{1}(\sigma)>0$, and two constants $\bar{\omega}, q$ such that: for all $\lambda$ with $\operatorname{Re} \lambda>\bar{\omega}$ we have that $\left(A_{n}-\lambda I\right)^{-1}$ exists as a linear map taking $X_{n}$ onto $X_{n}$ and

$$
\left\|\left(A_{n}-\lambda I\right)^{-1}\right\| \leqslant M_{1}(\operatorname{Re} \lambda)|\lambda-\bar{\omega}|^{q} .
$$

Remark. Stability implies weak stability because of the equivalent forms (2.15), (2.16a).

Once one has introduced such a "resolvent condition" for stability or weak stability one naturally turns to the Laplace transform (see Hille and Phillips [22]) as a tool of analysis (see Strang and Fix [41], Cerutti and Parter [8] for applied examples). Unfortunately, this approach seems to demand deeper results for the steady state problems. On the other hand, we are able to obtain "convergence theorems" for the time dependent problem in this weaker setting.

In particular, we consider an extension of Theorem $\mathrm{T}$ to the case of systems. We shall sometimes require the validity of a theorem of the following form: 
THEOREM S, N. Consider the steady state system of equations

$$
\left\{\begin{array}{l}
A \Phi_{N}=f_{N}, \\
A \Phi_{m}=\Phi_{m+1}+f_{m}, \quad m=0,1, \ldots, N-1,
\end{array}\right.
$$

and the related discrete system

$$
\left\{\begin{array}{l}
A_{n} \phi_{N}=P_{n} f_{N} \\
A_{n} \phi_{m}=P_{n}\left(\phi_{m+1}+f_{m}\right)=\phi_{m+1}+P_{n} f_{m}, \quad m=0,1, \ldots, N-1 .
\end{array}\right.
$$

There is a Banach space $Y \subset \widetilde{X}$ with $\|y\|_{\widetilde{X}} \leqslant C_{1}\|y\|_{Y}$ and

$$
\left\|\phi_{m}-\Phi_{m}\right\| \leqslant C_{2} F(n)\left\|\Phi_{m}\right\|_{Y} .
$$

Remark. It is perhaps worth noting that the Example 4 of Section 3 has the following properties:

(i) if $n>2 N$, then Theorem $\mathrm{S}, \mathrm{N}$ is valid,

(ii) the semidiscrete method is not weakly stable.

Before proceeding with the technical details of the arguments to come, it is perhaps worthwhile to sketch our approach.

Let $u(t)$ and $u_{n}(t)$ be the solution of (2.1) and (2.11), respectively. Consider their Laplace Transforms

$$
\hat{u}(s)=\int_{0}^{\infty} e^{-s t} u(t) d t, \quad \hat{u}_{n}(s)=\int_{0}^{\infty} e^{-s t} u_{n}(t) d t .
$$

These functions then satisfy

$$
\begin{aligned}
s \hat{u}(s) & =A \hat{u}+\hat{f}(s)+\hat{u}_{0}, \\
s \hat{u}_{n}(s) & =A_{n} \hat{u}_{n}+P_{n} \hat{f}(s)+\hat{U}_{0, n} .
\end{aligned}
$$

If we imagine $s$ fixed, then (5.7a) is a steady state problem similar to (2.4) which is solvable by virtue of the resolvent condition (2.5). Moreover, $(5.7 \mathrm{~b})$ is a discretization of this problem based on the same subspaces $X_{n}$ and the projections $P_{n}$. Thus, if an appropriate Theorem $\mathrm{T}(s)$ holds we would have an estimate of the form (for sufficiently smooth $\hat{u}(s)$ )

$$
\left\|\hat{u}(s)-\hat{u}_{n}(s)\right\| \leqslant C(s) F(n)\|\hat{u}(s)\|_{\widetilde{X}} .
$$

Applying the well-known integral inversion formula [50], we have with $s=\gamma+i \sigma$

$$
u(t)-u_{n}(t)=\frac{1}{2 \pi i} \int_{\gamma-i \infty}^{\gamma+i \infty} e^{s t}\left[\hat{u}(s)-\hat{u}_{n}(s)\right] d s,
$$

or

$$
\left\|u(t)-u_{n}(t)\right\| \leqslant \frac{e^{\gamma t}}{2 \pi} \int_{\gamma-i \infty}^{\gamma+i \infty}|C(s)| F(n)\|\hat{u}(s)\|_{\widetilde{X}}|d s| .
$$

Unfortunately, one must worry about a few technical details. In particular, there is the question of the convergence of the integrals in (5.9a), (5.9b). At this point, it is 
worth noting that this question is really very different in these two cases. The integral appearing in (5.9a) is the usual integral of complex variable theory, the Cauchy limit as the interval of integration tends to $\infty$. Moreover, the term $e^{i \sigma t}$ enables one to employ (directly or indirectly) the Riemann-Lebesgue Lemma to aid in this convergence. The integral appearing in (5.9b) is a Lebesgue integral, and its absolute convergence is required.

We require one technical lemma concerning the inversion formula (5.9a).

LEMMA 5.1. Let $v(t)$ satisfy the appropriate growth conditions so that its Laplace transform

$$
\hat{v}(s)=\int_{0}^{\infty} e^{-s t} v(t) d t
$$

exists for $\operatorname{Re} s>\bar{\omega}$. Let $k$ be an integer $>0$, and let $\gamma>\bar{\omega}$. Let $\tau>0$ be fixed, and suppose that $t<\tau$. Then

$$
\int_{\gamma-i \infty}^{\gamma+i \infty} e^{s t}\left[\frac{1}{s^{k}} \int_{\tau}^{\infty} e^{-s \sigma} v(\sigma) d \sigma\right] d s=0
$$

Proof. This result is an immediate consequence of the usual formal formulas for the Laplace transform and its inverse; see [50]. Intuitively, it asserts that the future cannot affect the present.

Remark. The growth conditions required in this lemma are easily verified in the applications to follow. For example, if $v(t)$ satisfies an equation of the form (2.1), then the appropriate estimates follow from the representation formula (2.2) and estimates on $f(s)$.

Instead of using Eqs. (5.7a), (5.7b), (5.9a), (5.9b) directly, we proceed as follows. Assume that $U_{0, n} \in X_{n}$ has been chosen so that we need only consider a related function $v(t)$, which satisfies

$$
v_{0}=V_{0, n}=0
$$

and,

$$
\|v(\cdot, s)\| \leqslant \frac{1}{|s|^{N+1}}
$$

for an appropriate choice of $N$.

Let

$$
W_{n}(s)=Q_{n} \hat{v}(s)
$$

Then, using (5.7a) (with $v_{0}=0$ ) we have, for an appropriate $\hat{g}(s)$,

$$
A_{n} W_{n}=P_{n} A \hat{v}(s)=P_{n}[s \hat{v}(s)-\hat{g}(s)]
$$

We rewrite $(5.12 \mathrm{a})$ as

$$
\left(s-A_{n}\right) W_{n}=P_{n}\left[\hat{g}(s)+s\left(W_{n}-\hat{v}(s)\right)\right]
$$


From (5.7b) we have

$$
\left(s-A_{n}\right) \hat{v}_{n}=P_{n}[\hat{g}(s)]
$$

Thus

$$
\left(s-A_{n}\right)\left[W_{n}-\hat{v}_{n}\right]=s P_{n}\left(W_{n}-\hat{v}(s)\right) .
$$

We may rewrite $(5.13 a)$ as

$$
\left[W_{n}(s)-\hat{v}_{n}(s)\right]=s\left(s-A_{n}\right)^{-1} P_{n}\left(Q_{n}-I\right) \hat{v}(s) .
$$

Our first result is a special case in which the "mild instability" is truly mild.

THEOREM 5.1. Suppose H.1 and H.2. Suppose the semidiscrete method is weakly stable with

$$
-1<q<0 .
$$

Suppose Theorem $\mathrm{T}$ holds, $u_{0} \in D(A) \cap \widetilde{X}$ and $U_{0, n}=Q_{n} U_{0}$. Furthermore, if $v(t)=$ $u(t)-u_{0}$, we suppose that $v(t), v^{\prime}(t), v^{\prime \prime}(t) \in D(A) \cap \widetilde{X}$ and satisfy the approximate conditions so that Lemma 5.1 applies. Then, there is a constant $K$ such that

$$
\begin{aligned}
&\left\|Q_{n} v(t)-v_{n}(t)\right\| \leqslant F(n) \cdot K e^{(\bar{\omega}+1) t}\left[\left\|\frac{d v}{d t}(0)\right\|_{\tilde{X}}+\max _{0 \leqslant \sigma \leqslant t}\left\|\frac{d^{2}}{d t^{2}} v(\sigma)\right\|_{\tilde{X}}\right] \\
&\left\|v(t)-v_{n}(t)\right\| \leqslant F(n)\|v(t)\|_{\tilde{X}}+\left\|Q_{n} v(t)-v_{n}(t)\right\| \\
&\left\|u(t)-u_{n}(t)\right\| \leqslant\left\|v(t)-v_{n}(t)\right\|+F(n)\left\|u_{0}\right\|_{\tilde{X}}
\end{aligned}
$$

Proof. Let $v_{n}=u_{n}-U_{n, 0}$; then

$$
u(t)-u_{n}(t)=v(t)-v_{n}(t)+\left(u_{0}-Q_{n} u_{0}\right)
$$

Thus, since Theorem T holds, we need only study $v(t)-v_{n}(t)$. We have

$$
\begin{aligned}
& \frac{d v}{d t}=A v+f(t)+A u_{0}, \quad v(0)=0, \\
& \frac{d v_{n}}{d t}=A_{n} v_{n}+P_{n}\left[f(t)+A u_{0}\right], \quad v_{n}(0)=0 .
\end{aligned}
$$

Applying the Laplace transform to these equations, we have

$$
s \hat{v}=A \hat{v}+\hat{g}, \quad s \hat{v}_{n}=A_{n} \hat{v}_{n}+P_{n} \hat{g},
$$

where $\hat{g}(s)=\hat{f}(s)+A u_{0} / s$.

Applying the inversion formula [50], we have with $s=(\bar{\omega}+1)+i \sigma$,

$$
Q_{n} v(t)-v_{n}(t)=\frac{1}{2 \pi i} \int_{(\bar{\omega}+1)-i \infty}^{(\bar{\omega}+1)+i \infty} e^{i s t}\left[W_{n}(x)-\hat{v}_{n}(s)\right] d s
$$

Applying (5.13b) and (5.2), we have

(5.16) $\left\|Q_{n} v(t)-v_{n}(t)\right\| \leqslant e^{(\bar{\omega}+1) t} F(n) \int_{\sigma=-\infty}^{\sigma=+\infty} M_{1}(\bar{\omega}+1)|s-\bar{\omega}|^{q}|s|\|\hat{v}(s)\|_{\tilde{X}} d \sigma$. 
Since $v(0)=0$, we have

$$
\hat{v}(s)=\frac{1}{s^{2}} \frac{d v}{d t}(0)+\frac{1}{s^{2}} \int_{0}^{\infty} e^{-s t}\left[\frac{d}{d t}\right]^{2} v(t) d t .
$$

Let

$$
\alpha=(1+|q|) / 2
$$

and let

$$
K=\frac{M_{1}(\bar{\omega}+1)}{2 \pi} \int_{-\infty}^{\infty} \frac{d \sigma}{\left(\sigma^{2}+1\right)^{\alpha}} .
$$

Then, applying Lemma 5.1 and (5.9b), we have (5.15a). The estimates (5.15b), (5.15c) follow from Theorem $\mathrm{T}$ and the triangle inequality together with the identities

$$
\begin{gathered}
u(t)-u_{n}(t)=v(t)-v_{n}(t)+\left(u_{0}-Q_{n} u_{0}\right), \\
\left(\frac{d}{d t}\right)^{k} u(t)=\left(\frac{d}{d t}\right)^{k} v(t) .
\end{gathered}
$$

Remark. The error estimate (5.15a) should be compared with (4.3b) of Theorem 4.1. If $f(t) \equiv 0$, the estimate (4.3b) depends only on $\|u(t)\|_{\widetilde{X}}$ and $\|d u / d t\|_{\widetilde{X}}$, while (5.12) also includes a term $\left\|d^{2} u / d t^{2}\right\|_{\tilde{X}}$. This last term is (apparently) not introduced because of the weak stability (as opposed to stability) but is rather due to the Laplace transform approach-see the remarks following (5.9a), (5.9b). However, the resolvent estimate with $-1<q<0$ is a very strong estimate-a sort of "weak holomorphic semigroup stability." Such an estimate should not be expected unless $T(t)$ is itself a holomorphic semigroup. This would occur if (2.1) were a parabolic problem. In fact, it is this estimate that was exploited by Cerutti and Parter [8]

Remark. While the results of Section 4 also seem to be based on the choice of $U_{0, n}=Q_{n} u_{0}$, there is a significant difference between those results and the result above. The stability assumption of Section 4 allows for an immediate result for any $U_{0, n}$ close to $u_{0}$. In the theorem above we are definitely limited to a restricted choice of $U_{0, n}$. This aspect of the theory will be very clearly emphasized in the more general result which follows.

Definition 5.2. Suppose Theorem S, N holds. Suppose

$$
\Phi_{j}=\frac{d^{j} u}{d t^{j}}(0) \in D(A) \cap \widetilde{X} \cap Y, \quad j=0,1, \ldots, N
$$

Then

$$
\left\{\begin{array}{l}
A \Phi_{N} \equiv f_{N} \\
A \Phi_{m}=\Phi_{m+1}-\left(\frac{d}{d t}\right)^{m} f(0), \quad m=0,1, \ldots, N-1
\end{array}\right.
$$


Let $\phi_{j}(n), j=0,1, \ldots, N$, be the solution of the corresponding discrete system

$$
\left\{\begin{array}{l}
A_{n} \phi_{N}(n)=P_{n} f_{N} \\
A_{n} \phi_{m}(n)=\phi_{m+1}(n)-P_{n}\left[\left(\frac{d}{d t}\right)^{m} f(0)\right], \quad m=0,1, \ldots, N-1 .
\end{array}\right.
$$

Then, we let $\widetilde{Q}_{n}$ be the operator which maps $u \rightarrow \phi_{0}(n)$, i.e.

$$
\widetilde{Q}_{n} u=\phi_{0}(n) .
$$

THEOREM 5.2. Suppose H.1 and H.2 hold. Suppose the semidiscrete method is weakly stable with $q \geqslant 0$. Suppose Theorem $\mathrm{T}$ holds and Theorem $\mathrm{S}, \mathrm{N}$ holds with

$$
N>q+1 \text {. }
$$

Suppose (5.19) holds and

$$
U_{0, n}=\widetilde{Q}_{n} u=\phi_{0}(n)
$$

Suppose

$$
\Phi_{j}=\frac{d^{j} u}{d t^{j}}(0) \in D(A) \cap \widetilde{X} \cap Y, \quad j=0,1, \ldots, N
$$

and

$$
d^{N+1} u / d t^{N+1} \in \tilde{X}
$$

and satisfies the necessary growth conditions so that Lemma 5.1 applies.

Then, there is a constant $K$ so that

$$
\left\|u(t)-u_{n}(t)\right\| \leqslant K e^{(\bar{\omega}+1) t} \max _{0 \leqslant \sigma \leqslant t}\left\|\left(\frac{d}{d t}\right)^{N+1} u(\sigma)\right\|_{\tilde{X}} F(n)
$$

$$
+C_{2} F(n) \sum_{j=\varnothing}^{N}\left\|\Phi_{j}\right\|_{Y} \frac{t^{j}}{j !}
$$

Proof. Let

$$
\begin{gathered}
v(t)=u(t)-\sum_{j=0}^{N} \Phi_{j} \frac{t^{j}}{j !} \\
v_{n}(t)=u_{n}(t)-\sum_{j=0}^{N} \phi_{j}(n) \frac{t^{j}}{j !} .
\end{gathered}
$$

Then a direct calculation shows that

$$
\text { (5.27a) } \quad \frac{d v}{d t}=A v+t^{N} \psi(t), \quad\left(\frac{d}{d t}\right)^{j} v(0)=0, \quad j=0,1, \ldots, N
$$




$$
\frac{d v_{n}}{d t}=A_{n} v_{n}+P_{n}\left(t^{N} \psi(t)\right), \quad\left(\frac{d}{d t}\right)^{j} v_{n}(0)=0, \quad j=0,1, \ldots, N,
$$

where $\psi(t)$ is determined from the Taylor series expansion of $f(t)$ and $u(t)$ and $A \Phi_{N}$.

Since Theorem $\mathrm{S}, \mathrm{N}$ holds we have

$$
\begin{aligned}
\left\|u(t)-u_{n}(t)\right\| & \leqslant\left\|v(t)-v_{n}(t)\right\|+\sum_{j=0}^{N} \frac{t^{j}}{j !}\left\|\phi_{j}(n)-\Phi_{j}\right\| \\
& \leqslant\left\|v(t)-v_{n}(t)\right\|+C_{2} F(n) \sum_{j=0}^{N}\left\|\Phi_{j}\right\|_{Y} \frac{t^{j}}{j !}
\end{aligned}
$$

Therefore, we need only study $\left\|v-v_{n}\right\|$.

The theorem now follows from (5.13b) as in Theorem 5.1.

Remark. Perhaps it seems very artificial to suggest such special initial values. However, such choices have already appeared in the literature. For example, in Cerutti and Parter [8] just this choice was made in order to assure the "superconvergence" at the knots. Working on the same problem, Dupont and Douglas [15] employed an even more complicated algorithm to obtain an appropriate initial value. See [14], [32] also.

6. Parabolic Problems in One Dimension. Let $\bar{X}=C[0,1]$, and let $A$ be given by

$$
\begin{gathered}
\text { domain }(A)=\left\{v \in C^{2}[0,1] ; v(0)=v(1)=0\right\}, \\
A u=\frac{d}{d x} a(x) \frac{d u}{d x}-b(x) \frac{d u}{d x}-c(x) u,
\end{gathered}
$$

where $a(x), b(x) \in C^{\prime}[0,1], c(x) \in C[0,1]$ and there exist constants $a_{0}, a_{1}, c_{0}, c_{1}$, such that

$$
0<a_{0} \leqslant a(x) \leqslant a_{1}, \quad 0 \leqslant c_{0} \leqslant c(x) \leqslant c_{1} .
$$

Let

$$
B=\max \{|b(x)|, 0 \leqslant x \leqslant 1\} .
$$

We consider the parabolic partial differential equation

$$
\left\{\begin{array}{l}
\rho(x) \frac{\partial u}{\partial t}=A u+r(x, t), \\
u(x, 0)=u^{0}(x),
\end{array}\right.
$$

where $\rho(x) \in C[0,1]$ and there are constants $\rho_{0}, \rho_{1}$ such that

$$
0<\rho_{0} \leqslant \rho(x) \leqslant \rho_{1} .
$$

Remark. The introduction of $\rho(x)$ has almost no effect on the analysis of Section 5. However, since the problems naturally arise in the form (6.5), we choose to include it in our work of this section. 
Consider a Ritz-Galerkin approach to this problem. That is, let $M_{N}$ be a sequence of subspaces of $\stackrel{\circ}{H}_{1}$ such that

$$
\text { dimension } M_{N}=N
$$

The Ritz-Galerkin approach to the numerical solution of the steady-state problem

$$
A y+r(x)=0
$$

is: find $Y(x) \in M_{N}$ such that

$$
\left(a Y^{\prime}, v^{\prime}\right)+\left(b Y^{\prime}, v\right)+(c Y, v)=(r, v)
$$

for every $v \in M_{N}$, where we use the notation

$$
(\phi, \psi)=\int_{0}^{1} \phi(x) \bar{\psi}(x) d x, \quad\|\phi\|_{L^{2}}^{2}=(\phi, \phi) .
$$

Our concern here is the establishment of the estimate (5.2) with $q=1 / 2$ and $M_{1}(\operatorname{Re} \lambda)=M_{1}$, a constant.

We first obtain some estimates for the special case when $b(x) \equiv 0$.

LEMma 6.1. Consider the eigenvalue problem. Find $\Phi(x, N) \in M_{N}$ and $\lambda$, a complex number, with $\Phi(x, N) \not \equiv 0$ such that

$$
\left(a \Phi^{\prime}, v^{\prime}\right)+(c \Phi, v)=\lambda(\rho \Phi, v)
$$

for every $v \in M_{N}$. Then the eigenvalues $\lambda_{j}, j=1,2, \ldots, N$, are real and satisfy

$$
\lambda_{j} \geqslant \frac{\pi^{2}}{a_{0}} j^{2}
$$

Moreover, the corresponding eigenfunctions $\Phi_{j}(x, N)$ may be chosen to satisfy the modified orthogonality condition

$$
\left(\rho \Phi_{j}, \Phi_{k}\right)=\delta_{j k}
$$

Proof. The inequality (6.11a) follows from the variational characterization of the eigenvalues and the minimax principle. The relationship (6.11b) follows from a standard argument about selfadjoint operators.

Lemma 6.2. Let $f(x) \in L^{2}(0,1)$, and let

$$
D \equiv\{s=\alpha+i \beta ;|\beta| \geqslant 1-\alpha\} .
$$

Let $s \in D$. Then there is a unique function $Y(x ; s) \in M_{N}$ which satisfies

$$
\left(a Y^{\prime}, v^{\prime}\right)+(c Y, v)+s(\rho Y, v)=(f, v)
$$

for every $v \in M_{N}$. Moreover,

$$
\|Y(\cdot, s)\|_{L^{2}} \leqslant \frac{\sqrt{2}}{\rho_{0}} \frac{1}{|s|}\|j\|_{L^{2}},
$$


148

SEYMOUR V. BARTER

$$
\left\|Y^{\prime}(\cdot, s)\right\|_{L^{2}} \leqslant \frac{M_{1}}{|s|^{1 / 2}}\|f\|_{L^{2}},
$$

where

$$
M_{1}=\left\{\frac{2}{a_{0} \rho_{0}}\left[1+\frac{\rho_{1}}{\rho_{0}}\right]\right\}^{1 / 2} .
$$

Proof. Since (6.6) holds, we may write

$$
f(x)=F(x) \rho(x)=\rho(x) \sum_{k=1}^{N} f_{k} \Phi_{k}(x, N),
$$

where

$$
f_{k}=\left(f, \Phi_{k}\right)
$$

We write

$$
Y(x)=\sum_{j=1}^{N} y_{j} \Phi_{j}(x)
$$

and find that

$$
\sum_{j=1}^{N} y_{j}\left(\lambda_{j}+s\right)\left(\rho \Phi_{j}, v\right)=\sum_{k=1}^{N} f_{k}\left(\rho \Phi_{k}, v\right)
$$

i.e.

$$
y_{j}=f_{j} /\left(\lambda_{j}+s\right) .
$$

Consider two cases. If $s=\alpha+i \beta$ and $\alpha \leqslant 0$, we have $|\beta| \geqslant 1-\alpha \geqslant|\alpha|$. Hence $2 \beta^{2} \geqslant|s|^{2}$ or $1 /|\beta| \leqslant \sqrt{2} /|s|$. Thus

$$
\frac{1}{\left|\lambda_{j}+s\right|} \leqslant \frac{1}{|\beta|} \leqslant \frac{\sqrt{2}}{|s|}, \quad \alpha \leqslant 0 .
$$

On the other hand, if $\alpha \geqslant 0$, then, since $\lambda_{j} \geqslant 0$,

$$
\frac{1}{\left|\lambda_{j}+s\right|} \leqslant \frac{1}{|s|}, \quad \alpha \geqslant 0 .
$$

Thus,

$$
(Y, Y) \leqslant \frac{1}{\rho_{0}}(\rho Y, Y)=\frac{1}{\rho_{0}}\left(\sum y_{j} \rho \Phi_{j}, \sum y_{k} \Phi_{k}\right),
$$

i.e.

$$
(Y, Y) \leqslant \frac{1}{\rho_{0}} \sum_{j=1}^{N}\left|y_{j}\right|^{2} \leqslant \frac{2}{\rho_{0}|s|^{2}} \sum_{j=1}^{N}\left|f_{j}\right|^{2} .
$$


However, $f_{j}=\left(f, \Phi_{j}\right)=\left(\rho F, \Phi_{j}\right)$. Hence,

$$
\sum\left|f_{j}\right|^{2} \leqslant(\rho F, F)=(f, f / \rho) \leqslant \frac{1}{\rho_{0}}\|f\|_{L^{2}}^{2} .
$$

Thus, (6.14a) is established. From this estimate we see that $Y(x)$ exists and is unique because $Y(x)$ is obtained as the solution of $N$ equations in $N$ unknowns.

In (6.13) we let $v=Y$. We have

$$
\left(a Y^{\prime}, Y^{\prime}\right)+(c Y, Y)+s(\rho Y, Y)=(f, Y)
$$

Thus

$$
a_{0}\left\|Y^{\prime}\right\|_{L^{2}}^{2} \leqslant\|f\|_{L^{2}} \cdot\|Y\|_{L^{2}}+|s| \rho_{1}\|Y\|_{L^{2}}^{2}
$$

Using (6.14a), we have

$$
a_{0}\left\|Y^{\prime}\right\|_{L^{2}}^{2} \leqslant \frac{\sqrt{2}}{\rho_{0}} \frac{1}{|s|}\|f\|_{L^{2}}^{2}+\frac{\rho_{1}}{\rho_{0}^{2}} \frac{2}{|s|}\|f\|_{L^{2}}^{2},
$$

which proves $(6.14 \mathrm{~b})$.

LEMMA 6.3. Let

$$
M_{2}=2 B M_{1}
$$

and let

$$
D_{1} \equiv D \cap\left\{s ;|s|>M_{2}^{2}\right\} \text {. }
$$

Suppose $r(x, s)$ is given and for every $s \in D_{1}$, as a function of $x, r(x, s) \in L^{2}[0,1]$. Let $s \in D_{1}$. Then, there is a unique solution $Y(x, s) \in M_{N}$ of the Ritz-Galerkin equations

$$
\left(a Y^{\prime}, v^{\prime}\right)+\left(b Y^{\prime}, v\right)+(c Y, v)+s(\rho Y, v)=(r, v)
$$

for every $v \in M_{N}$.

Moreover,

$$
\begin{aligned}
\|Y\|_{L^{2}} & \leqslant \frac{2 \sqrt{2}}{|2|}\|r(\cdot, s)\|_{L^{2}}, \\
\left\|Y^{\prime}(\cdot, s)\right\|_{L^{2}} & \leqslant \frac{2 M_{1}}{|s|^{1 / 2}}\|r(\cdot, s)\|_{L^{2}}, \\
\|Y\|_{\infty} & \leqslant \frac{2 M_{1}}{|s|^{1 / 2}}\|r(\cdot, s)\|_{\infty} .
\end{aligned}
$$

Proof. We assume that we have a solution $Y(x, s)$ and write

$$
G(x, s)=r(x, s)-b(x) Y^{\prime}(x, s) .
$$

Then, since $s \in D \subset D_{1}$, we have 


$$
\left\|Y^{\prime}(\cdot, s)\right\|_{L^{2}} \leqslant \frac{M_{1}}{|s|^{1 / 2}}\|G(\cdot, s)\|_{L^{2}},
$$

i.e.

$$
\left\|Y^{\prime}(\cdot, s)\right\|_{L^{2}} \leqslant \frac{M_{1}}{|s|^{1 / 2}}\|r(\cdot, s)\|_{L^{2}}+\frac{B M_{1}}{|s|^{1 / 2}}\left\|Y^{\prime}(\cdot, s)\right\|_{L^{2}} .
$$

Since $s \in D_{1}$, we have (6.21b). Applying (6.14a), we have

$$
\|Y(\cdot, s)\|_{L^{2}} \leqslant \frac{\sqrt{2}}{\rho_{0}} \frac{1}{|s|}\left[1+\frac{2 B M_{1}}{|s|^{1 / 2}}\right]\|r(\cdot, s)\|_{L^{2}},
$$

which implies (6.21a). Finally, (6.21c) follows from a standard inequality.

COROLlary. Let $\bar{\omega}=1+M_{2}^{2}$. Then the resolvent inequality (5.2) is satisfied with

$$
q=-1 / 2
$$

and

$$
M_{1}(\operatorname{Re} \lambda)=2 M_{1}
$$

Proof. See (6.21c).

Remark. Of course, the estimates of Lemma 6.3 imply stability in the $L^{2}$ norm. The force of this corollary is that we are obtaining the desired estimate in the maximum norm.

Application. In [47] and [48] M. F. Wheeler studied these problems. Essentially following her notation we let $\Delta=\left\{x_{j}\right\}_{j=0}^{M}$, where $0=x_{0}<x_{1}<\cdots<x_{M}$ $=1$. Let $I_{j}=\left(x_{j-1}, x_{j}\right), h_{j}=x_{j}-x_{j-1}, h=\max h_{j}$. Let $d$ be a fixed integer. Let

$$
M^{d, \Delta} \equiv\left\{v \in C^{0}[0,1] ; v \in P_{d}\left(I_{j}\right), j=1,2, \ldots, M\right\},
$$

where $P_{d}(I)$ is the set of polynomials of degree less than $d+1$ on an interval $I \subset \mathbf{R}$.

Let

$$
M_{0}^{d, \Delta} \equiv\left\{v \in M^{d, \Delta} ; v(0)=v(1)=0\right\} .
$$

The dimension of $M_{0}^{d, \Delta}$ is $M d-1$. Let $N=M d-1$ and set $M_{N}=M_{0}^{d, \Delta}$. A major result of [47] is, if $y \in W^{d+1, \infty}[0,1]$, then

$$
\left\|\left(I-Q_{N}\right) y\right\|_{\infty}=\left\|y-A_{N}^{-1} P_{N} A y\right\| \leqslant C h^{d+1}\|y\|_{W^{s+1, \infty}},
$$

where $P_{N}$ denotes $L^{2}$ projection onto $M_{N}$.

Thus, with $\widetilde{X}=W^{d+1, \infty}[0,1]$ we may apply Theorem 5.1 (if $\rho \neq \equiv 1$, we merely repeat the argument of Theorem 5.1. There is no difficulty with this modification) to obtain the following result for the parabolic problem (6.5).

THEOREM 6.1. Let $U(x, t) \in M_{N}$, for each fixed $t$, be that function which satisfies

$$
\left(\rho U_{t}, v\right)+\left(a U_{x}, v_{x}\right)+\left(b U_{x}, v\right)+(c U, v)=(r, v)
$$


for every $v \in M_{N}$, with

$$
U(x, 0)=U^{0}(x) \in M_{N}
$$

chosen as the solution of

$$
\begin{aligned}
\left(a U_{x}^{0}, v_{x}\right)+ & \left(b U_{x}^{0}, v\right)+\left(c U^{0}, v\right)+\bar{\omega}\left(\rho U^{0}, v\right) \\
& =\left(a u_{x}^{0}, v_{x}\right)+\left(b u_{x}^{0}, v\right)+\left(c u_{x}^{0}, v\right)+\bar{\omega}\left(\rho u^{0}, v\right)
\end{aligned}
$$

for every $v \in M_{N}$. Let $u(x, t)$ be the solution (6.5). Then,

$$
\begin{aligned}
\|U(t)-u(t)\|_{\infty} \leqslant & C h^{d+1}\left\|u^{0}\right\|_{\tilde{X}} \\
& +C h^{d+1} e^{(\bar{\omega}+1) t}\left\{\operatorname{Max}_{0 \leqslant \sigma<t}\left\|\frac{d u}{d t}\right\|_{\tilde{X}}+\underset{0<\sigma<t}{\operatorname{Max}}\left\|\frac{d^{2} u}{d t^{2}}\right\|_{\tilde{X}}\right\} .
\end{aligned}
$$

We observe that the result given in [48] is stronger because the error estimate given there does not depend on $\partial^{2} u / \partial t^{2}$. However, we have already discussed the occurrence of this term.

On the other hand, because we have obtained the estimate (5.2) (with $M_{1}(\operatorname{Re} \lambda)$ $=M_{1}$, a constant) in a region which reaches into the left halfplane, we easily obtain the next result.

THEOREM 6.2. With the same hypothesis as in Theorem 6.1 there is a constant $C(\tau)$ depending only on $\tau$, such that if $0<\tau \leqslant t$, then

$$
\|U(t)-u(t)\|_{\infty} \leqslant C h^{d+1}\left\|u^{0}\right\|_{\tilde{X}}+C(\tau) h^{d+1} e^{(\bar{\omega}+1) t}\left\{\operatorname{Max}_{0 \leqslant \sigma \leqslant t}\left\|\frac{d u}{d t}\right\|_{\tilde{X}}\right\}
$$

Proof. We turn to the proof of Theorem 5.1.

The estimate (5.17) is now replaced by the estimate

$$
\hat{v}(s)=\frac{1}{s} \int_{0}^{\infty} e^{-s t}\left[\frac{d}{d t} v(t)\right] d t
$$

Moreover, the contour of integration in the inversion formula (5.9a) is shifted from the vertical line $\operatorname{Real}(s)=\gamma$ to the curve $\Gamma$ given by: let $s=\alpha+i \beta$, then we have three straight lines

$$
\begin{cases}\beta=\alpha-2(\bar{\omega}+1), & -\infty<\alpha<(\bar{\omega}+1), \\ \alpha=\bar{\omega}+1, & -(\bar{\omega}+1) \leqslant \beta \leqslant(\bar{\omega}+1), \\ \beta=-\alpha+2(\bar{\omega}+1), & -\infty<\alpha<(\bar{\omega}+1) .\end{cases}
$$

As we see, the integrals now converge absolutely for $0<\tau \leqslant t$. See [8], [41] where similar computations are carried out. 
Finally, this contour can be used to obtain convergence $0<\tau \leqslant t$, when $U^{0}=$ $P_{N} u^{0}$; see [8]. Indeed, $A$-stability results are obtained by further shifts of the contour; see [8].

Department of Computer Sciences

University of Wisconsin

Madison, Wisconsin 53706

1. D. G. ARONSON, "On the correctness of partial differential operators and the von Neumann condition," Proc. Amer. Math. Soc., v. 14, 1963, pp. 948-955.

2. G. A. BAKER, J. H. BRAMBLE \& V. THOMÉE, "Single step Galerkin approximations for parabolic problems," Math. Comp., v. 31, 1977, pp. 818-847.

3. R. BEALS, "Laplace transform methods for evolution equations" in Boundary Value Problems for Linear Evolution Equations (Garnir, Ed.), D. Reidel Publishing Co., Dordrecht-Holland, 1977, pp. $1-26$.

4. R. BELLMAN, Stability Theory of Differential Equations, McGraw-Hill, New York, 1953.

5. C. DE BOOR \& B. K. SWARTZ, "Collocation at Gaussian points," SIAM J. Numer.

Anal., v. 10, 1973, pp. 582-606.

6. J. H. BRAMBLE \& V. THOMÉE, "Discrete time Galerkin methods for a parabolic boundary value problem," Ann. Mat. Pura Appl., v. 101, 1974, pp. 115-152.

7. J. H. BRAMBLE, A. H. SCHATZ, V. THOMÉE \& L. B. WAHLBIN, "Some convergence estimates for semidiscrete Galerkin type approximations for parabolic equations," SIAM J. Numer. Anal., v. 14, 1977, pp. 218-241.

8. J. H. CERUTTI \& S. V. PARTER, "Collocation methods for parabolic partial differential equations in one space dimension," Numer. Math., v. 26, 1976, pp. 227-254.

9. R. COURANT, K. FRIEDRICHS \& H. LEWY, "Über die partiellen Differenzengleichungen der mathematischen Physik," Math. Ann., v. 100, 1928, pp. 32-74.

10. MICHEL CROUZEIX, Sur l'Approximation des/Equations Différentielles Opérationnelles Linéaires par des Méthodes de Runge-Kutta, Thèse-AL', Université Paris VI, 1975.

11. J. DOUGLAS, JR., "On the relation between stability and convergence in the numerical solution of linear parabolic and hyperbolic differential equations," J. Soc. Indust. Appl. Math., v. 4, 1956, pp. 20-37.

12. J. DOUGLAS, JR., "On the numerical integration of $u_{x x}+u_{y y}=u_{t}$ by implicit methods," J. Soc. Indust. Appl. Math., v. 3, 1955, pp. 42-65.

13. J. DOUGLAS, JR. \& T. DUPONT, "Galerkin methods for parabolic equations," SIAM J. Numer. Anal., v. 7, 1970, pp. 575-626.

14. J. DOUGLAS, JR., T. DUPONT \& M. F. WHEELER, "A quasi-projection analysis of Galerkin methods for parabolic and hyperbolic equations," Math. Comp., v. 32, 1978, pp. 345362.

15. J. DOUGLAS, JR. \& T. DUPONT, Collocation Methods for Parabolic Equations in a Single Space Variable Based on $C^{1}$-Piecewise Polynomial Spaces, Lecture Notes in Math., Vol. 385 , Springer, New York, 1974.

16. H. FUJITA \& A. MIZUTANI, "On the finite element method for parabolic equations. I: Approximation of holomorphic semigroups," J. Math. Soc. Japan, v. 28, 1976, pp. 749-771.

17. G. FIX, "Effect of quadrature errors in finite element approximations of steady state, eigenvalues, and parabolic problems," Proc. of the O. N. R. Regional Symposium 1972 on the Mathematical Foundations of the Finite Element Method with Applications to Partial Differential Equations, Academic Press, New York, 1972.

18. G. FIX \& N. NASSIF, "On finite element approximations to time dependent problems," Numer. Math., v. 19, 1972, pp. 127-135.

19. G. E. FORSYTHE \& W. R. WASOW, Finite-Difference Methods for Partial Differential Equations, Wiley, New York, 1960.

20. MAX D. GUNZBURGER, "On the stability of Galerkin methods for initial-boundary value problems for hyperbolic systems," Math. Comp., v. 31, 1977, pp. 661-675.

21. H. P. HELFRICH, "Fehlerabschătzungen für das Galerkinverfahren zur Losung von Evolutionsgleichungen," Manuscripta Math., v. 13, 1974, pp. 219-235.

22. E. HILlE \& R. S. PHILliPS, Functional Analysis and Semigroups, Amer. Math. Soc. Colloq. Publ., Vol. 31, Amer. Math. Soc., Providence, R. I., 1957. 
23. F. JOHN, "On integration of parabolic equations by difference methods," Comm. Pure Appl. Math., v. 5, 1952, pp. 155-211.

24. M. L. JUNCOSA \& D. M. YOUNG, "On the order of convergence of solutions of a difference equation to a solution of the diffusion equation," J. Soc. Indust. Appl. Math., v. 1, 1953, pp. $111-135$.

25. M. L. JUNCOSA \& DAVID YOUNG, "On the convergence of a solution of a difference equation to a solution of the equation of diffusion," Proc. Amer. Math. Soc., v. 5, 1954, pp. 168174.

26. M. L. JUNCOSA \& DAVID YOUNG, "On the Crank-Nicolson procedure for solving parabolic partial differential equations," Proc. Cambridge Philos. Soc., v. 53, 1955, pp. 448-461.

27. H. O. KREISS, "Über die Losung des Cauchyproblems für lineare partielle Differentialgleichungen mit Hilfe von Differenzengleichungen," Acta Math., v. 101, 1959, pp. 179-199.

28. H. O. KREISS, "Über die Differenzapproximation höher Genauigkeit bei Anfangswertproblem für partielle Differentialgleichungen," Numer. Math., v. 1, 1959, pp. 186-202.

29. H. O. KREISS, "Über die Stabilitätsdefinition für Differenzengleichungen die partielle Differentialgleichungen approximieren," $B I T$, v. 2, 1962, pp. 153-181.

30. P. D. LAX \& R. D. RICHTMYER, "Survey of the stability of linear finite difference equations," Comm. Pure Appl. Math., v. 9, 1956, pp. 267-293.

31. W. LEUTERT, "On the convergence of unstable approximate solutions of the heat equation to the exact solution," J. Mathematical Phys., v. 30, 1952, pp. 245-251.

32. W. LEUTERT, "On the convergence of approximate solutions of the heat equation to the exact solution," Proc. Amer. Math. Soc., v. 2, 1951, pp. 433-439.

33. N. K. MADSEN \& R. F. SINCOVEC, "The numerical method of lines for the solution of nonlinear partial differential equations," Computational Methods in Nonlinear Mechanics (J. T. Oden et al., Eds.), Texas Institute for Computational Mechanics, Austin, Texas, 1974.

34. G. G. O'BRIEN, M. A. HYMAN \& S. KAPLAN, "A study of the numerical solution of partial differential equations," J. Math. and Phys., v. 29, 1951, pp. 223-251.

35. A. PAZY, Semigroups of Linear Operators and Applications to Partial Differential Equations, Lecture Note \#10, Univ. of Maryland, College Park, Maryland, 1974.

36. H. S. PRICE \& R. S. VARGA, "Error bounds for semidiscrete Galerkin approximations of parabolic problems with application to petroleum reservoir mechanics," Numerical Solution of Field Problems in Continuum Physics, SIAM-AMS Proceedings, Vol. II, Amer. Math. Soc., Providence, R. I., 1970, pp. 74-94.

37. P. A. RAVIART, "The use of numerical integration in finite element methods for solving parabolic equations," Conference on Numerical Analysis, Royal Irish Academy, Dublin, August 1972, Academic Press, New York, 1973.

38. R. D. RICHTMYER \& K. W. MORTON, Difference Methods for Initial Value Problems, 2nd ed., Interscience, New York, 1967.

39. V. S. RYABENKII \& A. F. FILIPPOW, Über die Stabilität von Differenzengleichungen, VEB Deutscher Verlag der Wissenschaften, Band 3, Berlin, 1960.

40. W. G. STRANG, "Difference methods for mixed boundary-value problems," Duke Math. $J .$, v. 27, 1960, pp. $221-232$.

41. G. STRANG \& G. J. FIX, An Analysis of the Finite Element Method, Prentice-Hall, Englewood Cliffs, N. J., 1973.

42. B. SWARTZ \& B. WENDROFF, "Generalized finite-difference schemes," Math. Comp., v. 23, 1969 , pp. 37-50.

43. H. F. TROTTER, "Approximation of semi-groups of operators," Pacific J. Math., v. 8, 1958 , pp. 887-919.

44. B. WENDROFF, "Well-posed problems and stable difference operators," SIAM J. Numer. Anal., v. 5, 1968, pp. 71-82.

45. M. F. WHEELER, "A priori $L_{2}$ error estimates for Galerkin approximations to parabolic partial differential equations," SIAM J. Numer. Anal., v. 10, 1973, pp. 723-759.

46. M. F. WHEELER, "A $C^{0}$ collocation-finite element method for two-point boundary problems and one space dimensional parabolic problems," SIAM J. Numer. Anal., v. 14, 1977, pp. $71-90$.

47. M. F. WHEELER, "An optimal $L_{\infty}$ error estimate for Galerkin approximations to solutions of two-point boundary value problems," SIAM J. Numer. Anal., v. 10, 1973, pp. 914-917.

48. M. F. WHEELER, " $L_{\infty}$ estimates of optimal orders for Galerkin methods for onedimensional second order parabolic and hyperbolic equations," SIAM J. Numer. Anal., v. 10, 1973, pp. $908-913$. 
49. V. THOMÉE, "Some convergence results for Galerkin methods for parabolic boundary value problems," Mathematical Aspects of Finite Elements in Partial Differential Equations (C. de Boor, Ed.), Academic Press, New York, 1974, pp. 55-58.

50. D. V. WIDDER, The Laplace Transform, Princeton Univ. Press, Princeton, N. J., 1946.

51. K. YOSIDA, Functional Analysis, Springer-Verlag, Berlin, 1965.

52. M. ZLÁMAL, Finite Element Multistep Methods for Parabolic Equations, ISNM 28, Birkhäuser Verlag, Basel and Stuttgart, 1975, pp. 177-187.

53. M. ZLÁMAL, "Finite element multistep discretizations of parabolic boundary value problems," Math. Comp., v. 29, 1975, pp. 350-359. 